\title{
MÉTODO TEMPO-INTENSIDADE: REVISÃO
}

NOEL G. CERQUEIRA JÚNIOR*

EVANILDA TEIXEIRA*

RENATA D. DE M.C. AMBONI ${ }^{\star * *}$

Este trabalho de revisão de literatura teve como objetivo apresentar informações sobre o método sensorial tempo-intensidade $(\mathrm{TI})$ necessárias ao desenvolvimento do protótipo de sistema computacional para coleta e tratamento de dados sensoriais de tempo-intensidade (TI-WEB). Além do histórico do desenvolvimento do método TI, desde a década de 50, foram abordadas as técnicas utilizadas na coleta e tratamento de dados, interpretação gráfica, análises estatísticas (ANOVA, ACP, STATIS) e modelos aplicados aos dados TI. Os estudos revisados neste trabalho evidenciaram o potencial do método tempo-intensidade para a avaliação dinâmica de diversos atributos sensoriais e a caracterização de alimentos e bebidas.

PALAVRAS-CHAVE: ANÁLISE SENSORIAL; ANÁLISE TEMPO-INTENSIDADE; CARACTERÍSTICA TEMPORAL.

* Mestre em Ciência e Tecnologia de Alimentos, Universidade Federal de Santa Catarina (UFSC), Florianópolis, SC (e-mail: noeljr@gmail.com).

** Doutora em Ciência e Tecnologia de Alimentos, Professora Titular, Departamento de Ciência e Tecnologia de Alimentos, UFSC, Florianópolis, SC.

*** Doutora em Química, Professora Adjunto, Departamento de Ciência e Tecnologia de Alimentos, UFSC, Florianópolis, SC. 


\section{INTRODUÇÃO}

Nos métodos sensoriais convencionais, como a análise descritiva quantitativa ou outras formas de avaliação utilizando escalas, as propriedades sensoriais sob investigação são consideradas implicitamente como fenômeno estático (DIJKSTERHUIS e PIGGOTT, 2001). Essa abordagem restringe a medida da resposta sensorial a apenas um ponto. Os julgadores fazem a integração de sua percepção em relação ao tempo para descrever valor de intensidade único (Equação 1). Tal processo resulta no mascaramento, ou perda total de informações relevantes (LEE III e PANGBORN, 1986). Assim, métodos que reconhecem as propriedades dinâmicas da ingestão de alimentos (mastigação e salivação), como o método tempo-intensidade (TI), produzem resultados mais válidos que os métodos estáticos (DIJKSTERHUIS e PIGGOTT, 2001).

$$
\text { resposta sensorial }=\int_{\text {tempo de ingestão }}^{\text {tempo até engolir } / \text { cuspir }}(\text { percepção }) d t
$$

As mudanças dinâmicas na intensidade das percepções são mais evidentes em alimentos que sofrem significativo derretimento, ou transformações de fase na mucosa oral. Em geral, os alimentos requerem manipulação seqüencial com a língua e mastigação para quebrar os sólidos e misturá-los com a saliva até ocorrer a deglutição (LEE III e PANGBORN, 1986).

Publicações com observações sobre as mudanças naturais na percepção do sabor apareceram na década de 30 , mas a metodologia tempo-intensidade ( $\mathrm{TI}$ ) só foi reconhecida como método sensorial na década de 50 (DIJKSTERHUIS e PIGGOTT, 2001). SÖSTRÖM e CAIRNCROSS (1953) utilizaram o método tempo-intensidade para avaliar o gosto residual amargo em diferentes cervejas e NEILSON (1957) empregou a mesma metodologia para avaliar o gosto de gomas de mascar, o amargor de cervejas e a redução do amargor em drogas. O registro dos dados era feito graficamente em papel com marcas de tempo e auxílio de cronômetro. MCNULTY (1974) introduziu sinais sonoros para indicar o momento da marcação da intensidade do estímulo percebido no papel, eliminando o potencial de distração do julgador com o cronômetro.

No final da década de 70 e início dos anos 80 vários sistemas eletromecânicos foram desenvolvidos para facilitar o desenho das curvas tempo-intensidade. LARSON-POWERS e PANGBORN (1978) desenvolveram sistema com papel de registro móvel, associado com barra metálica e escala não-estruturada na qual os julgadores apoiavam a caneta para expressar a intensidade percebida. SWARTZ (1980) simplificou esse método para avaliação de adoçantes sintéticos. Outros autores utilizaram sistema com disco potenciométrico conectado em registrador de papel contínuo (LAWLESS e SKINNER, 1979; BIRCH e OGUNMOYELA, 1980). Nesse momento, a análise manual das curvas tempo-intensidade ainda demandava grande esforço.

O desenvolvimento dos microcomputadores, na segunda metade da década de 80 , tornou possível a criação de sistemas de coleta de dados eletromecânicos em conjunto com programas específicos para a análise da informação tempo-intensidade. SCHMITT et al. (1984) utilizaram o sistema com papel de registro móvel para coletar os dados tempo-intensidade. Microcomputador acoplado em digitalizador mecânico foi usado para digitalizar os dados obtidos e transferí-los, através do modem, ao mainfraime para serem analisados mediante software de estatística (SAS).

GUINARD, PANGBORN e SHOEMAKER (1985) utilizaram joystick adaptado a uma escala e conectado ao microcomputador para gravar dados de intensidade de estímulos de gosto ao longo do tempo. O botão do joystick foi usado para o sinal de início e fim do teste e a tela para exibir as instruções. O sistema computadorizado mostrou-se mais rápido, mais acurado e mais interessante para os julgadores. YOSHIDA (1986) desenvolveu sistema em que a coleta de dados ocorria mediante mouse com movimentos limitados numa dimensão, acoplado a escala de magnitude. $\mathrm{O}$ autor utilizou alertas sonoros para indicar o início do teste e a ingestão da amostra. 
A evolução da informática nos anos 90 permitiu o surgimento de novos programas de computador amigáveis em substituição aos sistemas eletromecânicos de coleta de dados. Esses programas foram desenvolvidos de forma especializada, ou como parte de sistemas comerciais de coleta e análise de dados sensoriais (DIJKSTERHUIS e PIGGOTT, 2001). Alguns desses sistemas especializados foram desenvolvidos de forma independente ou por encomenda de laboratórios de pesquisa, que necessitavam do método tempo-intensidade e ao mesmo tempo não dispunham de recursos para a aquisição de sistema completo para análise sensorial (CARDELLO, 1996). De forma geral, o modelo de interface gráfica dos sistemas de coleta de dados tempo-intensidade desenvolvidos são semelhantes. Apresentam na tela o botão de início/fim do teste e escala de intensidade rotulada, composta de cursor deslizante atrelado ao movimento do mouse (HARKER; LAU e GUNSON, 2003; CARDELLO et al., 2003; BROWN, GÉRAULT e WALKELING, 1996).

DUIZER, BLOOM e FINDLAY (1995) realizaram estudo comparativo entre os dados de curvas tempo-intensidade para a percepção do gosto doce, obtidas por meio de escalas de intensidade verticais ou horizontais. Os resultados mostraram que a orientação da escala, na tela, não influenciou a resposta dos julgadores. A partir desse estudo, DUIZER, BLOOM e FINDLAY (1997) propuseram a adição de mais uma escala de intensidade, permitindo a análise simultânea de dois atributos sensoriais.

Alguns autores continuam a desenvolver pesquisas utilizando métodos manuais e eletromecânicos para coletar dados tempo-intensidade. KING e DUINEVELD (1999) utilizaram o sistema transdutor especial, equipamento que ao ser pressionado transmite a medida da força mecânica da mão para um amplificador, sendo os dados de tempo-intensidade coletados e gravados mediante software específico. VALENTOVÁ et al. (2002) realizaram experimentos comparativos entre o sistema manual com cartões de registro e sistemas computadorizados, usando a tela do computador e operando o mouse ou monitor sensível ao toque através de escala gráfica nãoestruturada. Nenhuma diferença quanto a dependência temporal foi observada entre os resultados obtidos pelo sistema manual e pelo procedimento computadorizado, operado em conjunto com o mouse. Também não houve diferença significativa entre esse último e o método com monitor sensível ao toque.

CARDELLO et al. (2003) destacaram o alto custo dos sistemas comerciais como empecilho para o desenvolvimento de pesquisas sensoriais de tempo-intensidade no Brasil. Para resolver tal problema, os autores desenvolveram o sistema de coleta de dados tempo-intensidade (SCDTI) que permitiu a realização de várias pesquisas usando essa metodologia (CARDELLO, SILVA e DAMÁSIO, 1999; CARDELLO e FARIA, 1999; SILVA et al., 2004).

O objetivo desta revisão foi apresentar informações sobre o método sensorial tempointensidade necessárias ao desenvolvimento do protótipo, baseado na tecnologia web, de sistema computacional para coleta e tratamento de dados sensoriais de tempo-intensidade. O protótipo, denominado TI-Web, ainda está em fase de testes e apresenta características relevantes como, armazenamento de dados de tempo-intensidade em base de dados, programação dos testes tempointensidade em etapa inicial para agilizar a realização dos testes, interface de coleta de dados tempo-intensidade amigável, obtenção de parâmetros das curvas tempo-intensidade, exportação de dados e desenho de curvas tempo-intensidade (CERQUEIRA JÚNIOR, 2005).

\section{INTERPRETAÇÃO E ANÁLISE DE CURVAS TI}

Antes do uso da informática, os recursos para a extração de informação das curvas tempointensidade eram limitados. A interpretação das curvas envolvia apenas a quantificação de pontos chaves ou parâmetros das curvas. Os parâmetros selecionados pelos pesquisadores eram, relativamente, os mais fáceis de serem extraídos. Intensidade máxima, tempo para intensidade 
máxima e tempo total foram os parâmetros utilizados com maior freqüência. O parâmetro área sob a curva, com muito esforço, era calculado manualmente (CLIFF e HEYMANN, 1993).

Com a informatização do método tempo-intensidade, parâmetros que exigiam cálculos mais complexos como a área sob a curva, taxa de crescimento e taxa de decrescimento da curva foram obtidos com maior facilidade (YOSHIDA, 1986; LALLEMAND et al., 1999). No entanto, nem todos os pesquisadores utilizavam a mesma terminologia e abreviações para descrever os parâmetros tempo-intensidade. A partir da revisão de CLIFF e HEYMANN (1993) foi estabelecida a nomenclatura de parâmetros tempo-intensidade para facilitar a interpretação da literatura. Os principais parâmetros e suas abreviações foram definidos como intensidade máxima (Imax), tempo para atingir a intensidade máxima (TImax), tempo total de duração do estímulo (Ttot), área total sob a curva (Área), tempo de duração da intensidade máxima (Platô), tempo antes do estímulo inicial (tempo de reação) (Tlag), tempo de leitura dos dados (Tfinal), taxa de crescimento (Mads), taxa de decrescimento (Mdes), área sob a curva antes da intensidade máxima (A), área sob a curva depois da intensidade máxima (B) e gosto residual calculado pela razão (A/B). A Figura 1 mostra curva típica tempo-intensidade de sensação única e alguns parâmetros que podem ser extraídos.

Muitos pesquisadores utilizaram a regressão linear para calcular a taxa de crescimento (Mads) e a taxa de decrescimento (Mdes) da curva. No entanto, alguns autores aproximaram o parâmetro taxa de crescimento a uma reta dividindo a intensidade máxima pelo tempo para atingir a intensidade máxima (SWARTZ, 1980).

As diferenças individuais entre os julgadores são únicas e reprodutíveis devido às diferenças na anatomia, na manipulação oral e de escala. Entretanto, essa informação é geralmente desprezada quando a interpretação dos parâmetros tempo-intensidade é realizada mediante análise de variância (ANOVA). A variância devida aos julgadores é removida e, então, torna-se possível a avaliação das diferenças entre as amostras para cada parâmetro tempo-intensidade. Tal forma de tratamento dos dados permite a reconstrução de curvas tempo-intensidade com os valores médios dos parâmetros (SWARTZ, 1980; SCHMITT et al., 1984; GUINARD, PANGBORN e SHOEMAKER, 1985).

\section{FIGURA 1 - CURVA TÍPICA TEMPO-INTENSIDADE COM ALGUNS PARÂMETROS REPRESENTADOS}

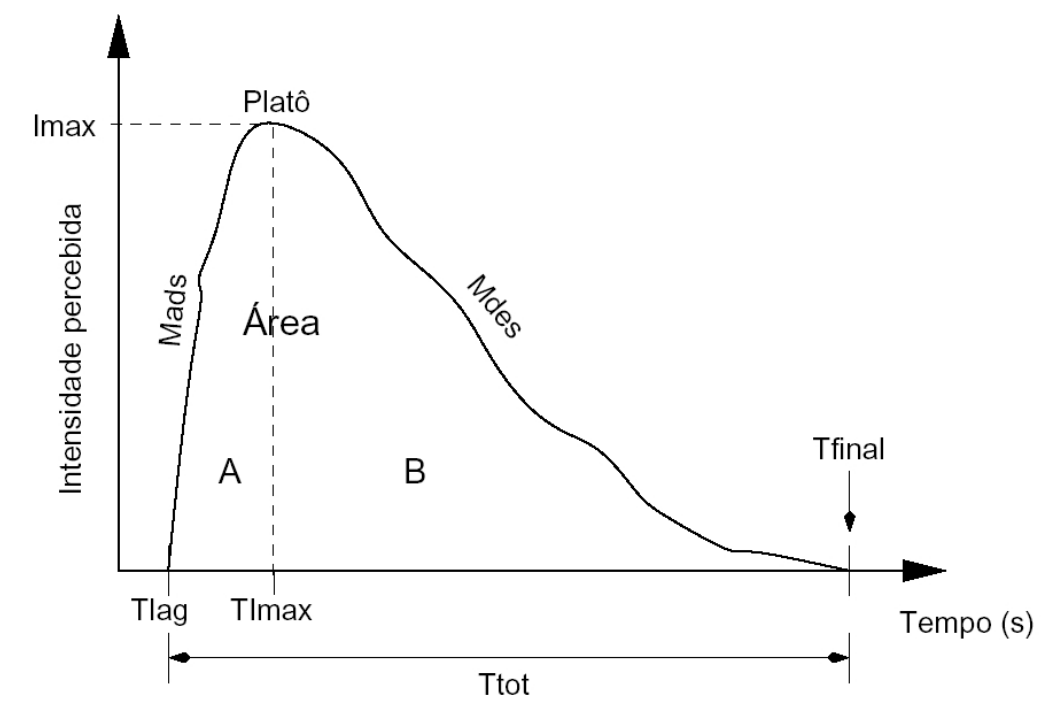

Valor de intensidade máxima (Imax), tempo para atingir a intensidade máxima (TImax), tempo total de duração do estímulo (Ttot), área total sob a curva (Área), tempo de duração da intensidade máxima (Platô), tempo antes do estímulo inicial (tempo de reação) (Tlag), tempo de leitura dos dados (Tfinal), taxa de crescimento (Mads), taxa de decrescimento (Mdes), área sob a curva antes da intensidade máxima (A), área sob a curva depois da intensidade máxima (B) (CLIFF e HEYMANN, 1993). 
Tentando caracterizar melhor as informações tempo-intensidade, OVERBOSCH, ENDEN e KEUR (1986) propuseram o cálculo de curvas médias pelas médias dos valores de intensidade em cada tempo. Nesse método, como as curvas de todos os julgadores são utilizadas para o cálculo da curva média, é desejável a concordância entre os resultados dos julgadores para evitar a distorção na forma da curva média em relação às curvas originais. As curvas obtidas apresentam mútiplos picos, mais largos ou mais estreitos. Esse problema pode ser parcialmente resolvido pela eliminação de algumas curvas individuais antes do cálculo da curva média, no entanto, a curva resultante não representa a média de todas as respostas. Para minimizar o problema das diferenças entre as curvas individuais, OVERBOSCH, ENDEN e KEUR (1986) propuseram a normalização dos dados do vetor intensidade. O cálculo das médias das intensidades, em todos os tempos para as partes ascendente e descendente da curva, separadas pelo TImax resultam na curva média. LIU e MACFIE (1990) propuseram modificação para o método de OVERBOSCH, ENDEN e KEUR (1986) por meio da normalização dos dados dos vetores nas direções do tempo e da intensidade. Tal modificação visou a eliminação dos problemas com a presença de platôs de intensidade máxima, de curvas que não terminassem com intensidade zero, da presença de valores de intensidade acidentais no final das curvas e a presença de curvas com múltiplos picos. Por esse método, os principais parâmetros da curva resultante correspondem aos parâmetros médios das curvas individuais (MACFIE e LIU, 1992).

VAN BUUREN (1992) sugeriu a utilização da análise de componentes principais (ACP) em curvas tempo-intensidade centradas para a criação de curvas principais de covariância. Isso permitiu que as informações comuns dos julgadores fossem extraídas com mais peso do que a informação devida aos desvios de resposta. As curvas construídas por esse método mostraram-se úteis para avaliação da equipe de julgadores, produzindo resultados mais representativos do que as curvas médias.

DIJKSTERHUIS (1993) propôs duas variações ao método de VAN BUUREN (1992) para o cálculo de curvas principais, baseadas no número de transformações iniciais dos dados coletados. A primeira alternativa sugere a centralização e normalização das curvas tempo-intensidade e aplicação da ACP. As curvas obtidas por esse processo são chamadas curvas principais de correlação e representam similaridades na forma geral das curvas tempo-intensidade. Outra alternativa envolve a aplicação da ACP sob as curvas originais sem tratamento inicial dos dados, sendo as curvas obtidas chamadas de curvas principais não-centradas. O primeiro componente principal não-centrado agregou melhor as características das curvas tempo-intensidade. O segundo componente principal não-centrado pode ser interpretado como modelo da taxa de crescimento e decrescimento das curvas tempo-intensidade (DIJKSTERHUIS, FLIPSEN e PUNTER, 1994).

DIJKSTERHUIS e BROEK (1995) sugeriram método para alongar ou encurtar os eixos de intensidade e de tempo das curvas tempo-intensidade. As escalas das curvas tempo-intensidade nesse método são inteiramente ajustadas de forma isotrópica, ou seja, a curva é alongada ou encurtada com o mesmo fator em ambas as direções. No entanto, as soluções fornecidas são incompletas porque não há razão para assumir que as escalas de intensidade e de tempo devam ser ajustadas na mesma proporção.

DIJKSTERHUIS e EILERS (1997) destacaram algumas desvantagens associadas com a utilização da ACP na análise de curvas TI. O aspecto ordinal do tempo é negligenciado na análise e qualquer permutação nos pontos de tempo resultam na mesma solução. O eixo do tempo não é representado na curva principal. Diante desses problemas, os autores encontraram a solução para permitir o ajuste das escalas de tempo e de intensidade de forma independente. Obtiveram a melhor curva aplicando modelo semi-paramétrico de curva prototipada projetada. Uma curva suave foi ajustada sobre os dados das escalas de intensidade e tempo separadamente para maximizar o ajuste das curvas tempo-intensidade individuais.

LALLEMAND et al. (1999) desenvolveram método para extração de parâmetros de curvas 
tempo-intensidade utilizando modelo trapezoidal. Esse modelo permite a extração das coordenadas de quatro pontos das curvas tempo-intensidade. Inicialmente são identificados os valores de intensidade máxima e do tempo para intensidade máxima correspondente da curva tempo-intensidade. Os pontos do trapézio são obtidos mediante relação com o valor da intensidade máxima. A primeira (A) e a última (D) coordenadas são extraídas dos valores de tempo correspondentes a 5\% da intensidade máxima. As outras duas coordenadas (B e C) são extraídas dos valores de 90\% da intensidade máxima. A partir dessas quatro coordenadas são calculados os parâmetros secundários, como a duração total do estímulo, área sob a curva, platô, taxa de crescimento e taxa de decrescimento da curva. O modelo funciona somente para curvas simples com apenas um pico.

PFEIFER, BOULTON e NOBLE (2000) desenvolveram modelo matemático geral para resposta ao estímulo doce. O modelo foi constituído pelo conjunto de equações diferenciais ordinárias (EDO), que expressam as taxas de difusão ao longo da fronteira da camada de saliva e adsorção e dessorção para as proteínas do sítio receptor nas células dos botões gustativos. Os valores iniciais para as equações foram obtidos pelas médias de dados TI observados. As curvas preditas pelo modelo mostraram-se próximas das respostas tempo-intensidade de 19 julgadores para diferentes adoçantes.

WENDIN, JANESTAD e HALL (2003) desenvolveram modelos baseados em equações polinomiais e equações diferenciais ordinárias (EDO) para serem ajustados aos dados experimentais de tempo-intensidade. Esses modelos foram utilizados para descrever e interpretar dados TI. Por meio das primeiras e segundas derivadas foi possível descrever as mudanças com o tempo da intensidade percebida e da área sob a curva. Os modelos EDO, quando obtidos de experimento bem modelado, permitem estimar a percepção dinâmica para ajustes experimentais dentro da escala dos ajustes originais.

CHAYA et al. (2003) investigaram a utilização do método Structuration des Tableaux A Trois Indices de la Statistique (STATIS) para a análise de curvas tempo-intensidade. O STATIS, método de análise de dados aplicável à matrizes de dados centrados, descreve o fenômeno tempodependente com as variáveis medidas ao longo do tempo em relação a grupo de indivíduos ou objetos. Adapta-se bem ao modelo de dados tempo-intensidade, tendo como variáveis os atributos sensoriais e como objeto o conjunto de produtos analisados. O estudo mostrou que o método STATIS é válido para a investigação da percepção dinâmica de características sensoriais.

EILERS e DIJKSTERHUIS (2004) propuseram modelo paramétrico genérico de cinco parâmetros para o ajuste de curvas TI. O modelo descreve a concentração da substância hipotética como o produto de curva em forma de "S" ascendente e outra curva em forma de "S" descendente. Supuseram que a resposta do julgador, gravada por instrumento, é proporcional ao logaritmo da concentração hipotética. A equação logística foi usada porque é uma das fórmulas mais simples para se obter a curva em forma de "S". O modelo para o ajuste de curvas TI comportou-se bem para curvas simples, mas não apresentou boa aproximação para curvas mais complexas com múltiplos platôs.

OVEJERO-LÓPEZ, BRO e BREDIE (2005) utilizaram diferentes métodos para analisar os dados de 800 curvas tempo-intensidade obtidas a partir de testes sensoriais com gomas de mascar para os atributos de gosto e sabor. Os métodos utilizados foram: a normalização de dados tempointensidade, proposta por LIU e MACFIE (1990), a extração de parâmetros ad hoc, a análise de componentes principais (ACP) e a análise de variância (ANOVA) com regressão parcial por mínimos quadrados. Além dos métodos citados, os autores propuseram mais dois métodos multivariados alternativos, aACP dupla e a análise paralela de fatores 2 . Todos os métodos possibilitaram interpretações dos dados tempo-intensidade e levaram a conclusões similares.

\section{APLICAÇOES DO MÉTODO TEMPO-INTENSIDADE}

A partir da década de 50, o método tempo-intensidade começou a ser utilizado com mais 
freqüência na análise sensorial de alimentos. Conforme o método foi sendo aperfeiçoado e informatizado, maior variedade de aplicações surgiram. Dentre as principais aplicações do método tempo-intensidade estão as pesquisas com edulcorantes, amargor em cerveja e características de textura em carne e frutas.

A aplicação do método tempo-intensidade na pesquisa com edulcorantes auxiliou a investigação de modelos teóricos para a percepção de gostos (BIRCH; LATYMER e HOLLAWAY, 1980; OVERBOSCH, 1986), a avaliação e quantificação do gosto residual (LARSON-POWERS e PANGBORN, 1978; LAWLESS e SKINNER, 1979; BIRCH e OGUNMOYELA, 1980; SWARTZ, 1980), o estudo dos efeitos sinergéticos ou supressores de combinações de edulcorantes (HARRISON e BERNHARD, 1984), o estudo das relações existentes entre as propriedades físicas e as características tempo-intensidade (CALVIÑO, 1986; PORTMANN, SERGHAT e MATHLOUTHI, 1992; WIET et al., 1993) e a caracterização de edulcorantes (MORI, YOTSUYANAGI e DOHI, 1994; CARDELLO, 1996; PORTMANN e KILCAST, 1996; CARDELLO, SILVA e DAMÁSIO, 1999).

O método tempo-intensidade foi empregado em parte da pesquisa realizada por CARDELLO (1996) para caracterização dos edulcorantes aspartame, ciclamato/sacarina (2:1) e estévia (CARDELLO, SILVA e DAMÁSIO, 1999). Foram utilizadas para o teste tempo-intensidade, soluções aquosas em equivalência de doçura com soluções de sacarose em concentrações variando de $3 \%$ a 50\% (p/v). A análise de variância (ANOVA) dos parâmetros das curvas tempo-intensidade e a análise de componentes principais (ACP) das médias dos parâmetros tempo-intensidade permitiram a caracterização dos edulcorantes nas concentrações analisadas.

CARDELLO, SILVA e DAMÁSIO (1998) realizaram a seleção de parâmetros tempointensidade para a descrição de características sensoriais temporais com relação a doçura e amargor dos edulcorantes aspartame, ciclamato/sacarina (2:1) e estévia. Para tanto, realizaram testes tempointensidade utilizando quatro concentrações equivalentes para cada produto. Os parâmetros tempointensidade extraídos das curvas foram tempo para atingir a intensidade máxima, tempo em que intensidade máxima começa a declinar, platô, área total sob a curva, tempo total de duração do estímulo e intensidade máxima percebida. Os parâmetros tempo-intensidade foram submetidos às análises de correlação e discriminante por passos, sendo verificado que o parâmetro área sob a curva poderia ser eliminado.

MAHAWANICH e SCHMIDT (2004) estudaram a relação entre a mobilidade da água e a doçura percebida em soluções de sacarose, frutose e glicose em concentrações de 0,15 mol/L até a saturação. Para medir a mobilidade da água utilizaram técnicas de ressonância magnética e a doçura percebida foi estudada pelo método tempo-intensidade. Os resultados revelaram ausência de correlação entre a mobilidade da água e a doçura percebida.

O método tempo-intensidade tem sido considerado como ferramenta muito útil na análise de bebidas com amargor característico, como cervejas (NEILSON, 1957; SCHMITT et al., 1984; VAN BUUREN, 1992; DIJKSTERHUIS, 1993) e a bebida de café (LEACH e NOBLE, 1986).

CARDELLO e FARIA (1999) utilizaram o método tempo-intensidade para estudar características de sabor de aguardente de cana envelhecida em barril de carvalho. Os resultados mostraram decréscimos na intensidade correlacionados com a duração dos sabores alcoólico e agressivo, e aumento no sabor de madeira durante quatro anos de maturação. Para o gosto doce houve pequeno aumento na intensidade após dois anos de maturação e elevação substancial na duração, correspondendo ao aumento da persistência do gosto doce sem elevação substancial em sua intensidade máxima.

KING e DUINEVELD (1999) pesquisaram as mudanças no amargor de cervejas envelhecidas naturalmente, utilizando o método tempo-intensidade em conjunto com a cromatografia a líquido de alta eficiência. A análise dos dados mostrou que os valores de intensidade máxima e área sob a curva tempo-intensidade diminuíram para o atributo amargor com a idade da cerveja e que a taxa de aumento da intensidade do gosto amargo foi maior em cervejas mais velhas. 
O método-tempo-intensidade foi utilizado por TECHAKRIENGKRAI et al. (2004) para pesquisar a percepção do amargor em 18 tipos de cerveja com diferentes concentrações de iso- $\alpha$ ácidos. Os resultados demonstraram a existência de correlação entre a concentração de iso- $\alpha$ ácidos e a percepção do gosto amargo. A comparação das curvas tempo-intensidade de 8 julgadores para um tipo de cerveja indicou diferenças entre os julgadores. No entanto, as curvas tempointensidade de um julgador para diferentes tipos de cerveja mostraram similaridades qualitativas e diferenças quantitativas.

SILVA et al. (2004) empregaram o método tempo-intensidade para avaliar o gosto amargo da bebida de café orgânico. Utilizaram 4 marcas comerciais de café orgânico (ORG-1, ORG-2, ORG-3, ORG-4) e uma de café convencional (CON). Os parâmetros da curva tempo-intensidade, tempo para a intensidade máxima, intensidade máxima, platô, área sob a curva, tempo correspondente ao ponto em que a intensidade máxima começa a declinar e tempo total de duração do estímulo foram submetidos à análise de variância (ANOVA), ao teste de comparação de médias (Duncan) e ACP. A marca ORG-3 apresentou maior intensidade máxima do gosto amargo em relação às demais marcas.

ISHIKAWA e NOBLE (1995) estudaram a interação entre a adstringência e a doçura em vinhos tintos pelo do método tempo-intensidade. Os parâmetros tempo-intensidade de intensidade máxima e duração total do estímulo para a adstringência decresceram significativamente com o aumento da concentração de sacarose. No entanto, nenhum parâmetro tempo-intensidade para a doçura foi afetado significativamente pelo nível de adstringência. Julgadores com baixo nível de salivação perceberam gosto residual adstringente mais persistente do que os julgadores com alto nível de salivação, indicando relação entre a salivação e a percepção do gosto residual amargo.

KALLITHRAKA et al. (2001) investigaram a relação entre a composição protéica da saliva e a percepção da adstringência do vinho tinto pelo método tempo-intensidade. Os resultados mostraram forte correlação entre os parâmetros tempo-intensidade e os valores de picos identificados mediante cromatografia a líquido de alta eficiência (CLAE). Os autores afirmaram que a concentração relativa de proteínas salivares são importantes para a percepção da adstringência.

VALENTOVÁ et al. (2002) utilizaram a metodologia tempo-intensidade para estudar o gosto adstringente do vinho, vermouth e outras bebidas. Os resultados mostraram que o parâmetro tempo de residência da amostra na boca influenciou os parâmetros de intensidade máxima e a taxa de decrescimento numa relação exponencial. As soluções de ácido tânico e de catequina apresentaram maior adstringência em água do que em vinho ou em suco de laranja.

O método tempo-intensidade tem sido usado para avaliar propriedades de textura em diversos produtos (LEE II, TAKAHASHI e PRUITT, 1992; DUIZER, GULLETT e FINDLAY, 1994). BRAXTON, DAUCHEL e BROWN (1996) utilizaram o método tempo-intensidade em conjunto com eletromiógrafo para estudar a influência da eficiência de mastigação na percepção da maciez de amostras de carne cozida. Os diferentes grupos de padrões de eficiência de mastigação evidenciaram tendências distintas quanto as formas das curvas tempo-intensidade para a maciez percebida, indicando discordância dos julgadores em relação aos conceitos de maciez da carne.

BROWN, GÉRAULT e WALKELING (1996) utilizaram o método tempo-intensidade para investigar os critérios usados por julgadores não-treinados em métodos sensoriais clássicos na avaliação da maciez e suculência de filés de carne bovina e suína. As formas das curvas tempointensidade para a maciez e suculência diferiram entre os julgadores, mas evidenciaram similaridades para os dois atributos individualmente. Alguns julgadores afirmaram que a suculência aumentou durante a mastigação. Os resultados indicaram que os julgadores diferiram em seus conceitos sensoriais de maciez e suculência. Os autores sustentaram o uso do método tempo-intensidade para a interpretação de diferenças individuais na percepção sensorial. BUTLER et al. (1996) também utilizaram o método tempo-intensidade para estudar a percepção de maciez de carne de porco em diferentes cortes. 
ZIMOCH e GULLETT (1997) avaliaram divergências temporais na percepção dos atributos suculência e maciez em filés de músculo bovino e diferenças entre as amostras de filé. A inspeção visual das curvas tempo-intensidade para a maciez permitiu reunir os julgadores em três grupos de acordo com padrões de eficiência de mastigação. O grupo maior foi composto pelos julgadores que mastigaram a carne com maior eficiência em curto espaço de tempo. O segundo grupo necessitou maior esforço para completar a mastigação e mais tempo para atingir a intensidade máxima. $\mathrm{O}$ último grupo foi composto pelos julgadores ineficientes na mastigação. A análise de componentes principais dos dados tempo-intensidade indicou boa homogeneidade da equipe sensorial, tanto para a maciez quanto para a suculência. Os autores encontraram correlações significativas entre a maciez e a suculência para os parâmetros tempo para a intensidade máxima e duração total do estímulo. Isto sugere que os padrões de mastigação podem exercer efeito na percepção sensorial dos atributos maciez e suculência.

DESOBRY-BANON e VICKERS (1998) determinaram a medida dinâmica da coesividade de massa, usando o método tempo-intensidade para amostras elaboradas em laboratório (a base de goma xantana 5, 10 e 20\%) e outras adquiridas comercialmente (massa para biscoito, queijo fatiado tipo americano, Thin Rolled Gold Pretzels). Os resultados permitiram identificar dois padrões característicos nas curvas tempo-intensidade. No primeiro, as respostas para coesividade mostraram que as amostras a base de goma xantana e a massa para biscoito atingiram rapidamente valor máximo próximo ao final da primeira manipulação com a língua e decresceu conforme o produto foi sendo quebrado por manipulações repetidas. O segundo, obtido das amostras de pretzel, apresentou intervalo de tempo maior até a intensidade máxima refletindo o tempo necessário para a absorção de saliva e a manipulação. Os parâmetros intensidade máxima, duração total do estímulo e área sob a curva foram os melhores descritores dos produtos.

SMITH e NOBLE (1998) utilizaram o método tempo-intensidade para investigar a influência do aumento da viscosidade na acidez e adstringência percebidas em soluções de sulfato de alumínio e ácido cítrico com adição de metil celulose. Os parâmetros de intensidade máxima e duração da adstringência percebida decresceram com o aumento da viscosidade, demonstrando o papel da viscosidade na redução da adstringência para os compostos estudados.

A suculência de frutas frescas (melancia, melão Honey Dew, morango, abacaxi e maçã), típicas da Nova Zelândia, foi avaliada por HARKER, LAU e GUNSON (2003) pela metodologia tempo-intensidade. Os resultados do estudo demonstraram que as frutas apresentam nível mais elevado de suculência do que outros alimentos, tal como a carne que é descrita (geralmente) como suculenta. Os parâmetros tempo-intensidade associados à melancia evidenciaram valores altos de intensidade máxima, porém baixos valores para a duração total do estímulo. Por outro lado, a maçã apresentou valores moderados de intensidade máxima e valores bem maiores para a duração total do estímulo que os da melancia. O estudo destacou o atributo suculência como bom descritor de muitas frutas e que a sensação de suculência muda de intensidade ao longo da mastigação.

LYNCH et al. (1993) estudaram o efeito dos óleos de girassol e de coco na percepção dos gostos básicos (doce, amargo, salgado e ácido) pelo método tempo-intensidade. Os julgadores avaliaram géis com gostos básicos após espalharem óleo na boca. Os resultados mostraram que os dois óleos reduziram a intensidade máxima e suprimiram as respostas de outras medidas de tempo-intensidade. O óleo de coco tendeu a exercer efeitos mais consistentes e supressivos do que o óleo de girassol.

WENDIN e HALL (2001) utilizaram o método tempo-intensidade em conjunto com a análise descritiva e estudo reológico para caracterizar a influência da gordura, espessante e emulsificante nas propriedades sensoriais de molho de salada. Os atributos sensoriais avaliados foram acidez, doçura e oleosidade. Verificaram que as medidas sensoriais dinâmicas e descritivas relacionaramse principalmente com o atributo oleosidade.

GUINARD, PANGBORN e SHOEMAKER (2002) mostraram que o aumento da concentração 
de gordura em molho de salada (emulsão óleo em água) provoca redução nos valores dos parâmetros intensidade máxima, tempo total de estímulo e área sobre a curva tempo-intensidade para os sabores de alho e picante e atraso na percepção do gosto ácido. Afirmaram que em alimentos como molhos de salada, tanto as interações moleculares com a fase lipídica quanto a estrutura física da matriz do alimento afetam as propriedades de liberação dos sabores.

SINESIO, MONETA e ESTI (2005) estudaram a percepção temporal dos atributos amargor e pungência em óleo de oliva varietal, armazenado durante período superior a dezoito meses pelo método tempo-intensidade. Observaram tendência de queda na intensidade e na duração dos atributos pungência e amargor com o armazenamento. $\mathrm{O}$ amargor foi o atributo dominante logo após a produção do óleo. Por outro lado, as amostras com maior tempo de armazenamento evidenciaram decréscimo rápido do amargor e a pungência tornou-se o atributo predominante (apesar de também decrescer).

LINFORTH, BAEK e TAYLOR (1999) utilizaram o método tempo-intensidade em conjunto com a técnica de ionização química da pressão atmosférica - espectroscopia de massa para estudar a liberação dos voláteis de géis durante a ingestão. As curvas tempo-intensidade para o atributo sabor apresentaram muita semelhança com as curvas obtidas mediante espectroscopia, indicando relação entre as mudanças na concentração de voláteis durante a ingestão e a percepção sensorial da intensidade dos voláteis. Afirmaram que para eventos rápidos de ingestão pode ocorrer atraso na percepção depois da concentração máxima do estímulo respiratório. Por outro lado, se o processo de ingestão for longo pode ocorrer adaptação sensorial.

MIETTINEN, HYVÖNEN e TUORILA (2003) utilizaram o método tempo-intensidade para estudar a percepção retronasal do aroma de composto polar (diacetil) e de composto não-polar (linalool) em leite desnatado com adição de óleo de canola (0, 1, 5 ou 10\%). Perceberam que a liberação temporal do linalool permite questionar, em parte, a indicação freqüentemente repetida que a redução de gordura resulta em liberação mais rápida do aroma.

No estudo de COURREGELONGUE, SCHLICH e NOBLE (1999), o método tempo-intensidade permitiu a observação de aumento na intensidade da adstringência em leite de soja avaliado por consumidores normais de cerveja. Evidenciaram que a adição de carboxi-metil-celulose e sacarose ao leite de soja promoveu redução na intensidade da adstringência.

\section{CONSIDERAÇÕES FINAIS}

Ao longo dos últimos 60 anos, o método tempo-intensidade vem se consolidando como importante ferramenta para a análise sensorial. A disponibilidade de vários sistemas computadorizados comerciais para análise sensorial, dando suporte ao método tempo-intensidade, permitiu melhora notável na coleta de dados, no cálculo e na interpretação dos dados de tempointensidade.

Os métodos de análise estatística multivariada têm se tornado populares e importantes nas pesquisas de análise sensorial. Existem várias publicações recentes com propostas de novos métodos de análise de dados multivariados e aperfeiçoamento dos métodos já estabelecidos, que auxiliam a interpretação dos dados sensoriais de tempo-intensidade.

A análise exploratória dos dados com o uso da análise multivariada permite revelar estruturas que podem auxiliar na interpretação dos resultados e na construção de hipóteses em pesquisas futuras.

Entre as aplicações do método tempo-intensidade revisadas neste artigo destacam-se: o estudo de gostos básicos para a caracterização de produtos como edulcorantes (doçura e amargor), cerveja (amargor) e bebida de café (amargor), o estudo da textura em carnes, frutas (maciez e suculência), gomas, massas (coesividade) e molhos de salada (oleosidade), o estudo da 
adstringência e do amargor em bebidas como, por exemplo, o vinho e o estudo de aromas (géis saborizados).

\section{ABSTRACT}

\section{TIME-INTENSITY METHOD - REVIEW}

The objective of this literature review work was to present information about the time-intensity (TI) sensorial method, necessary to the development of computerized time-intensity TI-WEB prototype which performed the collection and treatment of data. Besides the description of the development of the TI method since 1950, techniques for collection of data, interpretation and statistical analysis (ANOVA, PCA, STATIS) of time-intensity curves were approached. The reviewed studies have evidenced the potential of the time-intensity method in sensory dynamic analysis of several attributes of food and drinks.

KEY-WORDS: SENSORY ANALYSIS; TIME-INTENSITY ANALYSIS; TEMPORAL CHARACTERISTIC.

\section{REFERÊNCIAS}

1 BIRCH, G.; LATYMER, Z; HOLLAWAY, M. Intensity/time relationships in sweetness: evidence for a queue hypothesis in taste chemoreception. Chemical Senses, v. 5, n. 1, p. 63-78, 1980.

2 BIRCH, G.; OGUNMOYELA, G. Taste properties of cocoa drinks with an added bitter/sweet sugar: intensity/time effects. Journal of Food Technology, v. 15, p. 549-555, 1980.

3 BRAXTON, D.; DAUCHEL, C.; BROWN, W. E. Association between chewing efficiency and mastication patterns for meat, and influence on tenderness perception. Food Quality and Preference, v. 7, n. 3-4, p. 217-23, 1996.

4 BROWN, W. E.; GÉRAULT, S.; WALKELING, I. Diversity of perceptions of meat tenderness and juiciness by consumers: a time-intensity study. Journal of Texture Studies, v. 27, p. 475-492, 1996.

5 BUTLER, G. et al. Time-intensity as a tool for the measurement of meat tenderness. Food Quality and Preference, v. 7, n. 3-4, p. 193-204, 1996.

6 CALVIÑO, A. M. Perception of sweetness: the effects of concentration and temperature. Physiology \& Behavior, v. 36, p. 1021-1028, 1986.

7 CARDELLO, H.; SILVA, M.; DAMÁSIO, M. Seleção de parâmetros das curvas de tempo-intensidade de edulcorantes. In: CONGRESSO BRASILEIRO DE CIÊNCIA E TECNOLOGIA DE ALIMENTOS, 16, 1998, Rio de Janeiro. Anais...Rio de Janeiro: SBCTA, 1998. p. 384-387.

8 CARDELLO, H. M. A. B. Caracterização sensorial de aspartame, ciclamato/sacarina 2:1 e extrato de folhas de Estévia (Stevea rebaudiana Bertoni): equivalências em doçura, análise descritiva quantitativa e análise tempo-intensidade. Campinas, 1996. 237 f. Tese (Doutorado), Universidade Estadual de Campinas.

9 CARDELLO, H. M. A. B.; FARIA, J. B. Análise tempo-intensidade de características sensoriais de aguardente de cana durante o envelhecimento em tonel de carvalho (Quercus $s p$ ). Boletim da Sociedade Brasileira de Ciência e Tecnologia de Alimentos, v. 33, n. 1, p. 27-34, 1999.

10 CARDELLO, H. M. A. B.; SILVA, M. A. A. P. da; DAMÁSIO, M. H. Análise tempo-intensidade dos gostos doce e amargo de extrato de folhas de estévia (Stevea rebaudiana Bertoni) em doçura equivalente a sacarose. Ciência e Tecnologia de Alimentos, v. 19, n. 1, p. 8-14, 1999.

11 CARDELLO, H. M. A. B. et al. Programa sistema de coleta de dados tempo-intensidade - scdti. Boletim da Sociedade Brasileira de Ciência e Tecnologia de Alimentos, v. 37, p. 54-60, 2003.

12 CARDELLO, H. M. A. B.; SILVA, M. D.; DAMÁSIO, M. Measurement of the relative sweetness of stevia extract, aspartame and cyclamate/saccharin blend as compared to sucrose at different concentrations. Plant Foods for Human Nutrition, v. 54, n. 2, p. 119-129, 1999.

13 CERQUEIRA JÚNIOR, N. G. Desenvolvimento de um sistema computadorizado para a coleta e o tratamento de dados tempo-intensidade. Florianópolis, 2005. 57 f. Dissertação (Mestrado), Universidade Federal de Santa Catarina.

14 CHAYA, C. et al. Use of the statis method to analyze time-intensity profiling data. Food Quality and Preference, v. 15, p. 3-12, 2003.

15 CLIFF, M.; HEYMANN, H. Development and use of time-intensity methodology for sensory evaluation: a review. Food Research International, v. 26, p. 375-385, 1993. 
16 COURREGELONGUE, S.; SCHLICH, P.; NOBLE, A. C. Using repeated ingestion to determine the effect of sweetness, viscosity and oiliness on temporal perception of soymilk astringency. Food Quality and Preference, v. 10, p. 273-279, 1999.

17 DESOBRY-BANON, S.; VICKERS, Z. Cohesiveness of mass evaluation by time-intensity and single value measurements. Journal of Food Science, v. 63, n. 1, p. 174-176, 1998.

18 DIJKSTERHUIS, G.; VAN DEN BROEK, E. Matching the shape of time-intensity curves. Journal of Sensory Studies, v. 10, p. 149-161, 1995.

19 DIJKSTERHUIS, G.; FLIPSEN, M.; PUNTER, P. Principal component analysis of ti-curves: three methods compared. Food Quality and Preference, v. 5, n. 1-2, p. 121-127, 1994.

20 DIJKSTERHUIS, G. B. Principal component analysis of time-intensity bitterness curves. Journal of Sensory Studies, v. 8, n. 4, p. 317-328, 1993.

21 DIJKSTERHUIS, G. B.; EILERS, P. Modeling time-intensity curves using prototype curves. Food Quality and Preference, v. 8, n. 2 , p. $131-140,1997$.

22 DIJKSTERHUIS, G. B.; PIGGOTT, J. R. Dynamic methods of sensory analysis. Trends in Food Science \& Technology, v. 11, p. $284-290,2001$.

23 DUIZER, L.; GULLETT, E.; FINDLAY, C. The effect of masticatory patterns as measured by time-intensity and electromyography on the perception of bovine muscle tenderness. Journal of Sensory Studies, v. 9, p. 33-46, 1994.

24 DUIZER, L. M.; BLOOM, K.; FINDLAY, C. J. The effect of line orientation on the recording of time-intensity perception of sweetener solutions. Food Quality and Preference, v. 6, n. 2, p. 121-126, 1995.

25 DUIZER, L. M.; BLOOM, K.; FINDLAY, C. J. Dual-attribute time-intensity sensory evaluation: a new method for temporal measurement of sensory perceptions. Food Quality and Preference, v. 8, n. 4, p. 261-269, 1997.

26 EILERS, P. H.; DIJKSTERHUIS, G. B. A parametric model for time-intensity curves. Food Quality and Preference, v. 15, n. 3, p. 239-245, 2004.

27 GUINARD, J.X.; PANGBORN, R.; SHOEMAKER, C. Computerized procedure for time-intensity sensory measurements. Journal of Food Science, v. 50, p. 543-546, 1985.

28 GUINARD, J.X.; PANGBORN, R. M.; SHOEMAKER, C. F. Flavor release from salad dressing varying in fat and garlic flavor. Food Quality and Preference, v. 13, n. 3, p. 129-137, 2002.

29 HARKER, F. R.; LAU, K.; GUNSON, F. A. Juiciness of fresh fruit: a time/intensity study. Post harvest Biology and Technology, v. 29, p. 55-60, 2003.

30 HARRISON, S. K.; BERNHARD, R. A. Time-intensity sensory characteristics of saccharin, xylitol and galactose and their effect on the sweetness of lactose. Journal of Food Science, v. 49, p. 780-794, 1984.

31 ISHIKAWA, T.; NOBLE, A. C. Temporal perception of astringency and sweetness in red wine. Food Quality and Preference, v. 6, n. 1 , p. $27-33,1995$.

32 KALLITHRAKA, S. et al. Correlations between saliva protein composition and some t-i parameters of astringency. Food Quality and Preference, v. 12, n. 2, p. 145-152, 2001.

33 KING, B. M.; DUINEVELD, C. Changes in bitterness as beer ages naturally. Food Quality and Preference, v. 10, n. 4 5, p. 315-324, 1999

34 LALLEMAND, M. et al. Extracting parameters from time-intensity curves using a trapezoid model: the example of some sensory attributes of ice cream. Journal of Sensory Studies, v. 14, p. 387-399, 1999.

35 LARSON-POWERS, M.; PANGBORN, R. Paired comparison and time-intensity measurements of the sensory properties of beverages and gelatins containing sucrose or synthetic sweeteners. Journal of Food Science, v. 43, p. 41-46, 1978.

36 LAWLESS, H. T.; SKINNER, E. Z. The duration and perceived intensity of sucrose taste. Physiology \& Behavior, v. 25, n. 3 , p. $180-184,1979$.

37 LEACH, E. J.; NOBLE, A. C. Comparison of bitterness of caffeine and quinine by a time-intensity procedure. Chemical Senses, v. 11, n. 3, p. 339-345, 1986.

38 LEE III, W. E.; PANGBORN, R. M. Time-intensity: the temporal aspects of sensory perception. Food Technology, v. 40, p. 71-82, 1986.

39 LEE III, W. E.; TAKAHASHI, K.; PRUITT, J. Temporal aspects of the oral processing of viscous solutions. Food Technology, v. 46, p. $106-112,1992$. 
40 LINFORTH, R.; BAEK, I.; TAYLOR, A. Simultaneous instrumental and sensory analysis of volatile release from gelatin and pectin/gelatin gels. Food Chemistry, v. 65, p. 77-83, 1999.

41 LIU, Y. H.; MACFIE, H. J. H. Methods for averaging time-intensity curves. Chemical Senses, v. 15, n. 4, p. 471-484, 1990.

42 LYNCH, J. et al. A time-intensity study of the effect of oil mouth coatings on taste perception. Chemical Senses, v. 18, n. 2, p. 121-129, 1993.

43 MACFIE, H. J. H.; LIU, Y. H. Developments in the analysis of time-intensity curves. Food Technology, v. 46, p. 92-97, 1992.

44 MAHAWANICH, T.; SCHMIDT, S. J. Molecular mobility and the perceived sweetness of sucrose, fructose and glucose solutions. Food Chemistry, v. 84, p. 169-179, 2004.

45 MCNULTY, P. B. Intensity-time curves for flavored oil-in-water emulsions. Journal of Food Science, v. 39 , p. 55 57, 1974.

46 MIETTINEN, S.M.; HYVÖNEN, L.; TUORILA, H. Timing of intensity perception of a polar vs nonpolar aroma compound in the presence of added vegetable fat in milk. Journal of Agricultural and Food Chemistry, v. 57, p. 5437-5443, 2003.

47 MORI, E. E. M.; YOTSUYANAGI, K.; DOHI, C. T. Utilização da técnica tempo-intensidade na caracterização de doçura da sacarose e do esteviosídeo. Coletânea ITAL, v. 24, n. 2, p. 137-148, 1994.

48 NEILSON, A. J. Time-intensity studies. Drug and Cosmetic Industry, v. 80, n. 4, p. 452-3,534, 1957.

49 OVEJERO-LÓPEZ, I.; BRO, R.; BREDIE, W. L. P. Univariate and multivariate modeling of flavour release in chewing gum using time-intensity: a comparison of data analytical methods. Food Quality and Preference, v. 16, n. 4, p. $327-3432005$.

50 OVERBOSCH, P. A theoretical model for perceived intensity in human taste and smell as a function of time. Chemical Senses, v. 11, n. 3, p. 315-329, 1986.

51 OVERBOSCH, P.; ENDEN, J. van den; KEUR, B. An improved method for measuring perceived intensity/time relationships in human taste and smell. Chemical Senses, v. 11, n. 3, p. 331-338, 1986.

52 PFEIFER, J. F.; BOULTON, R. B.; NOBLE, A. C. Modeling the sweetness response using time-intensity data. Food Quality and Preference, v. 11, n. 12 , p. 129-138, 2000.

53 PORTMANN, M.O.; KILCAST, D. Psychophysical characterization of new sweeteners of commercial importance for the EC food industry. Food Chemistry, v. 56, n. 3, p. 291-302, 1996.

54 PORTMANN, M.O.; SERGHAT, S.; MATHLOUTHI, M. Study of some factors affecting intensity/time characteristics of sweetness. Food Chemistry, v. 44, p. 83-92, 1992.

55 SCHMITT, D. et al. An improved method for evaluating time-intensity data. Journal of Food Science, v. 49, p. 539-542, 1984.

56 SILVA, A. F. da et al. Avaliação do gosto amargo da bebida de café (Coffea arabica l.) orgânico por meio da análise tempo-intensidade. Ciência e Tecnologia de Alimentos, v. 24, n. 3, p. 468-472, 2004.

57 SINESIO, F.; MONETA, E.; ESTI, M. The dynamic sensory evaluation of bitterness and pungency in virgin olive oil. Food Quality and Preference, v. 16, n. 6, p. 557-564 2005.

$58 \mathrm{SMITH}$, A. K.; NOBLE, A. C. Effects of increased viscosity on the sourness and astringency of aluminum sulfate and citric acid. Food Quality and Preference, v. 9, n. 3, p. 139-144, 1998.

59 SÖSTRÖM, L. B.; CAIRNCROSS, S. E. The descriptive analysis of flavor food acceptance testing methodology. In: PERYAM, D.; PILGRIM, F.; PETERSON, M. (Ed.). Food Acceptance Testing Methodology. Symposium Palmer House. Chicago: Quartermaster Food and Container Institute, 1953.

60 SWARTZ, M. Sensory screening of synthetic sweeteners using time-intensity evaluations. Journal of Food Science, v. 45, n. 3, p. 577-581, 1980.

61 TECHAKRIENGKRAI, I. et al. Relationships of sensory bitterness in lager beers to iso- $\alpha$-acid contents. Journal of the Institute of Brewing, v. 110, n. 1, p. 51-56, 2004.

62 VALENTOVÁ, H. et al. Time intensity studies of astringent taste. Food Chemistry, v. 78, p. 29-37, 2002.

63 VAN BUUREN, S. Analyzing time-intensity responses in sensory evaluation. Food Technology, v. 46, n. 2, p. $101-104,1992$.

64 WENDIN, K.; HALL, G. Influences of fat, thickener and emulsifier contents on salad dressing: static and dynamic sensory 
and rheological analyses. Lebensmittel-Wissenschaft und -Technologie, v. 34, p. 222-233, 2001.

65 WENDIN, K.; JANESTAD, H.; HALL, G. Modeling and analysis of dynamic sensory data. Food Quality and Preference, v. 14, n. 8 , p. 663-671, 2003.

66 WIET, S. G. et al. Fat concentration affects sweetness and sensory profiles of sucrose, sucralose, and aspartame. Journal of Food Science, v. 58, n. 3, p. 599-602, 1993.

67 YOSHIDA, M. A microcomputer (pc 9801/ms mouse) system to record and analyse time-intensity curves of sweetness. Chemical Senses, v. 11, n. 1, p. 105-118, 1986.

68 ZIMOCH, J.; GULLETT, E. A. Temporal aspects of perception of juiciness and tenderness of beef. Food Quality and Preference, v. 8, n. 3, p. 203-211, 1997. 